\title{
Abusive head trauma: don't overlook bridging vein thrombosis
}

\author{
Catherine Adamsbaum • Caroline Rambaud
}

Received: 15 February 2012 /Revised: 14 April 2012 / Accepted: 1 May 2012 / Published online: 12 August 2012

(C) Springer-Verlag 2012

Few paediatric diagnoses generate as much controversy as abusive head trauma (AHT), also known as shaken baby/shaken impact syndrome (or SBS). Major signs such as multifocal subdural haematomas, cerebral injury, retinal haemorrhage and skeletal fractures are exhaustively described in myriad radiological reports, and are the result of extremely violent trauma. While bruises have significant diagnostic value, they may be missed when located in hidden areas like behind the ears and in the axilla. In some cases, subdural haematomas appear to be isolated, with no other apparent signs, making diagnosis difficult. We would like to stress the need for careful vertex screening to look for bridging vein thrombosis, which - as some pathologists have pointed out - has high diagnostic value [1].

The bridging veins are draining veins that arise from the coalescence of the superficial cortical venous net-

C. Adamsbaum $(\square)$

Pediatric Radiology Department, Bicetre Hospital, Assistance Publique-Hôpitaux de Paris and Paris Descartes University,

Paris, France

e-mail: adamsbaum.catherine@gmail.com

C. Rambaud

Hospital Anatomic Pathology and Forensic Department, Raymond Poincaré Hospital, Assistance Publique-Hôpitaux de Paris and Versailles-Saint Quentin en Yvelines University, Paris, France work at the midline, within the subarachnoid space. They extend, bridge-like, at intervals along the midline, from their attachment to the arachnoid at the medial border of the cerebral hemispheres to the superior sagittal sinus of the dura mater, into which they flow after having traversed the arachnoid space and the deep layers of the dura mater [2]. These veins constitute a short, non-tortuous, perpendicular pipeline between the

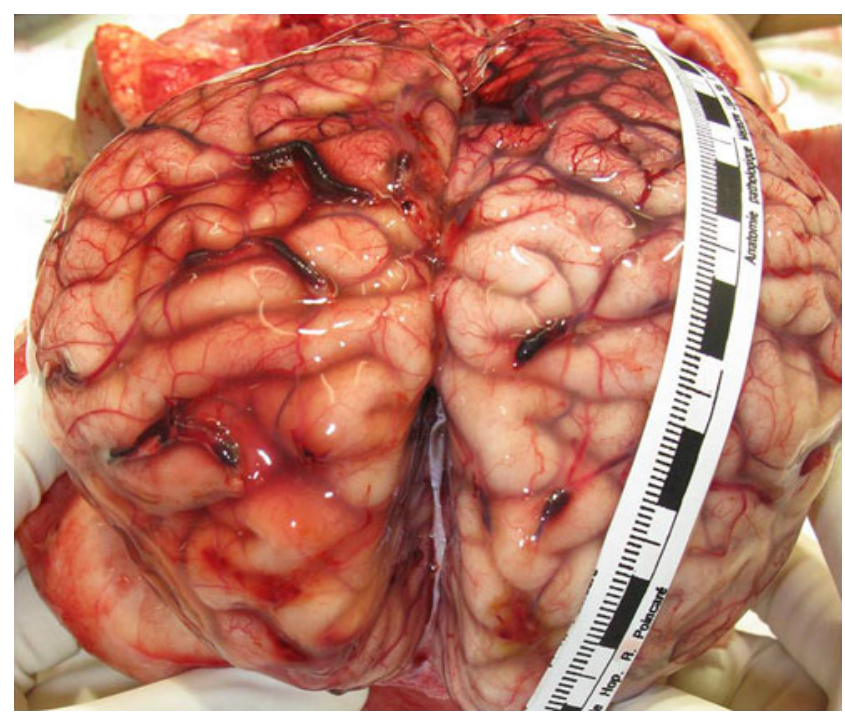

Fig. 1 Multiple bridging vein thrombosis in a case of confessed abusive head trauma (AHT) with subdural diffuse haematoma. Macroscopic view. The baby died after 12 days in the intensive care unit. No pre-mortem imaging available 

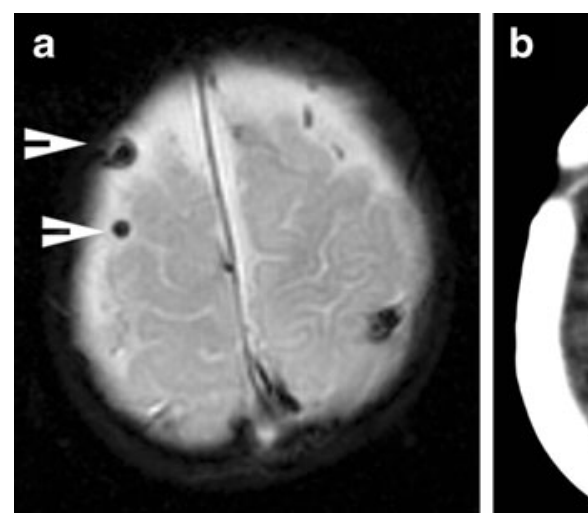

Fig. 2 Images in a 5-month-old boy presenting with seizures, loss of consciousness and multiple classic metaphyseal fractures. Probable multiple bridging vein thrombosis (arrowheads), seen on T2*

arachnoid and the dura, making them particularly vulnerable to rupture during anteroposterior movements of the brain within the cranium [1]. In the absence of a clotting disorder, such ruptures lead to thrombosis within a few hours (Fig. 1). However, standard autopsy procedures may damage the bridging veins and thereby the demonstration of their rupture and/or thrombosis is often difficult $[2,3]$.

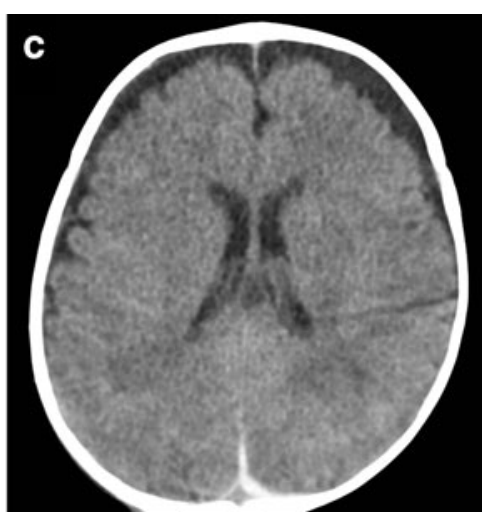

weighted axial MRI (a) and on axial CT (b). Chronic bifrontal subdural haematoma (hypodense) associated with hyperdense, acute interhemispheric subdural haematoma (c)

Anatomically, the subdural portion of the bridging veins is particularly fragile. The veins vary in number and diameter among individuals, with the diameter inversely proportional to the number [4]. These anatomical variations may explain why some individuals are more susceptible to injury than others with similar trauma. In any case, the existence of ruptured bridging veins confirms the traumatic nature of the subdural haematoma,
Fig. 3 A case of confessed AHT in a 4-month-old boy. Chronic bilateral subdural haematoma (hypodense) with acute thrombosis of a right bridging vein (arrowhead), i.e. hematoma of different ages. a, b nonenhanced axial CT, $\mathbf{c}$ T2*weighted coronal MRI. d MRI after gadolinium injection
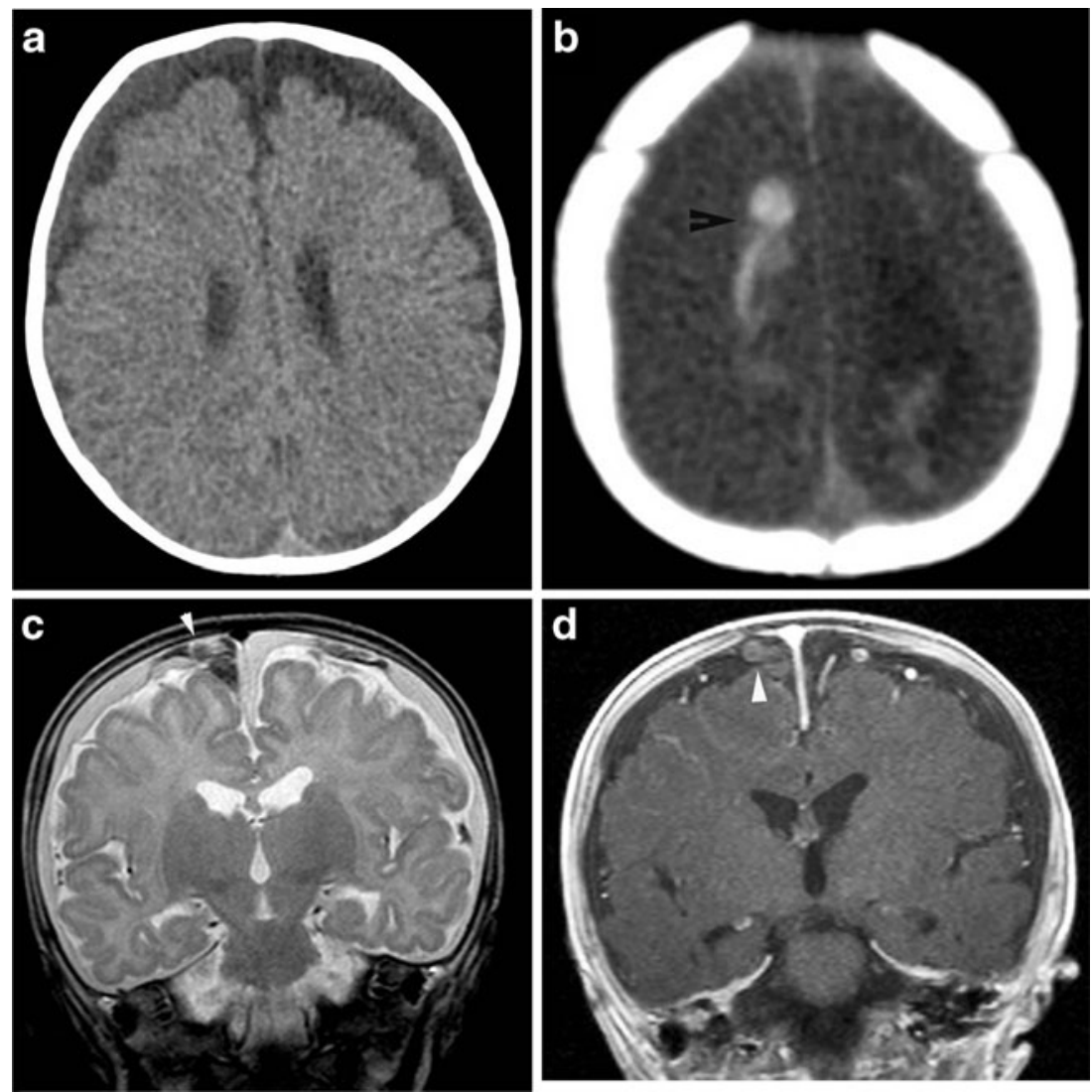
Fig. 4 A case of confessed AHT in a 7-month-old boy presenting with recurrent vomiting. Chronic bilateral subdural haematoma (hypodense) with acute thrombosis of left bridging veins (arrowhead), i.e. hematoma of different ages. a, b Axial CT images. c, d T2weighted axial MR images
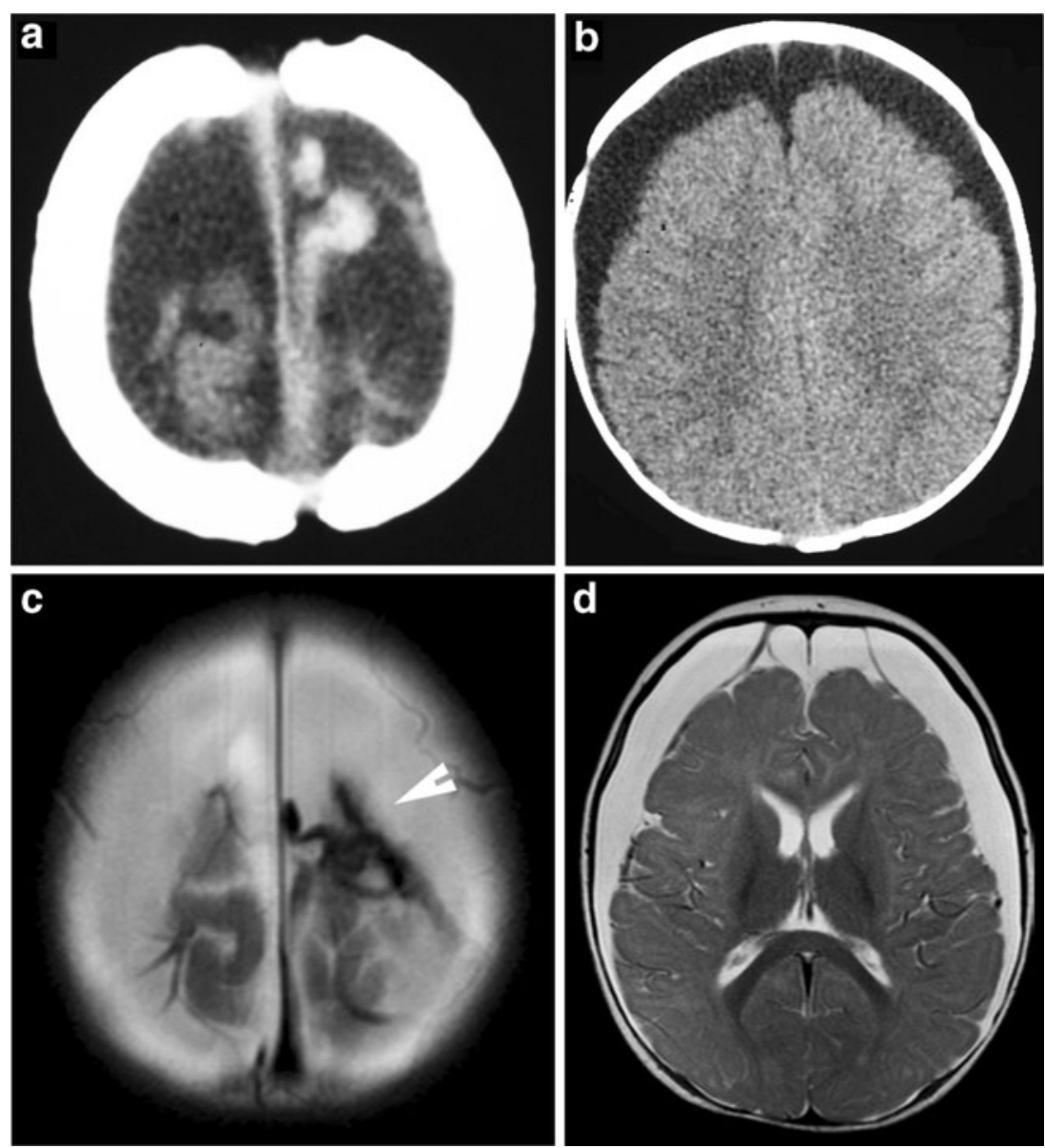

and in a non-accidental context offers a significant diagnostic element in favour of violent anteroposterior movements [5].

In some cases, analysis of the vertex by CT, or even better by MRI, may directly show clots with a tubular shape typically suggestive of acute bridging vein thromboses (Figs. 2, 3 and 4). Even though these tubular clots could also correspond to clotted blood in the subarachnoid space, they should be considered in any case as markers of acutely disrupted veins. Though rarely mentioned in the radiological literature, this sign is very important to recognise, given its crucial diagnostic value $[6,7]$. New susceptibility-weighted imaging (SWI) should help and, if in doubt, gadolinium injection - which is not typically administered in traumamay be used as a solving tool (Fig. 3).

Acknowledgments The authors thank C. Rey-Salmon, MD and B. Husson, MD for advises and Pascale Zerbini for manuscript preparation.

\section{References}

1. Yamashima T, Friede RL (1984) Why do bridging veins rupture into the virtual subdural space? J Neurol Neurosurg Psychiatry 47:121127

2. Squier W, Mack J (2009) The neuropathology of infant subdural haemorrhage. Forensic Sci Int 187:6-13

3. Maxeiner H (2001) Demonstration and interpretation of bridging vein ruptures in cases of infantile subdural bleedings. J Forensic Sci 46:85-93

4. Stein KM, Ruf K, Ganten MK et al (2006) Representation of cerebral bridging veins in infants by postmortem computed tomography. Forensic Sci Int 163:93-101

5. Ehrlich E, Maxeiner H, Lange J (2003) Postmortem radiological investigation of bridging vein ruptures. Leg Med 5(S1): S225-S227

6. Zimmerman RA, Bilaniuk LT, Farina L (2007) Non-accidental brain trauma in infants: diffusion imaging, contributions to understanding the injury process. J Neuroradiol 34:109-114

7. Adamsbaum C, Méjean N, Merzoug V et al (2010) How to explore and report children with suspected non-accidental trauma. Pediatr Radiol 40:932-938 